\title{
Manipulation and trapping of sub-micron bioparticles using dielectrophoresis
}

\author{
N.G. Green ${ }^{a}$, H. Morgan ${ }^{a}, *$ Joel J. Milner ${ }^{b}$

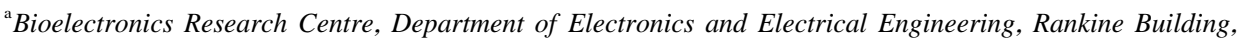 \\ Oakfield Avenue, University of Glasgow, Glasgow G12 8QQ, Scotland, UK \\ ${ }^{\mathrm{b}}$ Plant Molecular Science Group, Division of Biochemistry and Molecular Biology, Institute of Biological \\ and Life Sciences, University of Glasgow, Glasgow G12 8QQ, Scotland, UK
}

Received 14 May 1997; received in revised form 25 June 1997; accepted 23 July 1997

\begin{abstract}
A non-uniform alternating electric field induces motion in polarisable particles called dielectrophoresis. The effect is governed by the relative magnitudes of the dielectric properties of the medium and the particles. The technology has been used to manipulate particles for biotechnological applications, including purification, fractionation and concentration of cells and microorganisms. However, the lower size limit for the dielectrophoretic manipulation of particles was believed to be about $1 \mu \mathrm{m}$, but recent work has proved otherwise. The dielectrophoretic movement and properties of latex beads and a simple rod-shaped virus, tobacco mosaic virus (TMV), have been measured using microfabricated electrode structures. Measurements have been made over a range of suspending medium conductivities, applied frequencies and electric field strengths. It is shown that under appropriate conditions both latex beads and tobacco mosaic virus particles can be selectively attracted to regions of high electric field strength located at the tips of microfabricated electrode structures. The ability to selectively trap and separate bio-particles has many potential applications in the area of biotechnology. (C) 1997 Elsevier Science B.V.
\end{abstract}

Keywords: Latex spheres; Tobacco mosaic virus; Dielectrophoresis; Micro-electrodes; Bio-particle manipulation

\footnotetext{
*Corresponding author. Tel.: + 44141 3305237; fax: + 44141 3304907; e-mail h.morgan@elec.gla.ac.uk
} 


\section{Introduction}

The term dielectrophoresis (DEP) was first coined by Pohl in 1951 [1], to define the motion of polarisable particles (charged or uncharged) in non-uniform, alternating electric fields. Subsequently the subject of a monograph by this author [2], the field has recently developed into an important new aspect of biotechnology and has been recently reviewed in detail by Pethig [3]. In brief, the physical basis is that a particle polarises under the influence of an electric field: i.e. charges induced at the particle interface, together with intrinsic charges both in and on the particle are displaced by the application of the field. The magnitude and time-dependence of this displacement dictates the absolute force exerted on the particle. This force, called the dielectrophoretic force, depends on several parameters, namely the magnitude of the electric field and its non-uniformity, the volume of the particle and also a factor which is proportional to the difference between the polarisability (capacitance and conductance) of the particle and the medium. The force can be either positive or negative, depending on the relative magnitudes of these polarisabilities [3]. Movement of the particles to regions of high electric field strength is called positive DEP, whereas movement to regions of low electric field strength is called negative DEP. Since the polarisability of the particle is frequency dependent, the DEP force varies with frequency and it is this important factor which makes DEP a powerful means of separating dielectrically similar, but not identical particles. For applications in biology the electric fields are applied to the solution containing the particles via microfabricated electrode arrays [3].

Dielectrophoresis is a non-invasive and non-destructive technique and consequently has potential use in a wide range of biotechnological applications. Several recent publications have shown that the separation and concentration of a variety of microorganisms and mammalian cells is possible. Separation of bacteria and yeasts [4,5], normal and cancerous cells [6-8], together with separation and enrichment of CD34+ stem cells from bone marrow [9-11] has been described recently. These studies have shown the great potential for the application of DEP to biotechnology.

In general the DEP force depends on the electric field strength and the particle volume. Until recently, it was thought that dielectrophoretic separation and manipulation of bioparticles less than $1 \mu \mathrm{m}$ in size was impossible owing to the effects of Brownian motion [2]. At room temperature, this randomising force can be much larger than the force imparted on a particle by DEP and this problem increases as the volume of the particle is reduced. Therefore, in order to manipulate particles of the size of virions, large DEP forces must be generated and this requires high electric field strengths. For example, the DEP force necessary to move a cell or micro-organism is of the order of a few $\mathrm{pN}$ [12] which can be generated with an electric field of $1 \times 10^{5} \mathrm{~V} \mathrm{~m}^{-1}$ (equivalent to $10 \mathrm{~V}$ across an electrode gap of $0.1 \mathrm{~mm}$ ). However, in order to manipulate a virion of $100 \mathrm{~nm}$ in diameter a field of at least $5 \times 10^{6} \mathrm{~V} \mathrm{~m}^{-1}$, equivalent to $500 \mathrm{~V}$ across a 0.1 $\mathrm{mm}$ electrode gap, is required. Such voltages are not practical to generate at the field frequencies where DEP occurs and the only alternative method of producing the high field strengths is to reduce the electrode dimensions. For example, a voltage of $5 \mathrm{~V}$ across a $1 \mu \mathrm{m}$ gap will generate a suitable field strength of $5 \times 10^{6} \mathrm{~V} \mathrm{~m}^{-1}$.

The advent of new micro- and nano-fabrication methods in the semiconductor 
industry has meant that such small electrodes can now be manufactured with relative ease [13], opening the way to the DEP manipulation of latex beads, virions, DNA and protein molecules. This paper describes applications of DEP to the manipulation of both sub-micron sized latex particles and tobacco mosaic virus (TMV) particles.

\section{Materials and methods}

\subsection{Electrode fabrication}

Electrodes were manufactured on glass microscope slides $(25 \mathrm{~mm} \times 75 \mathrm{~mm})$ using standard photolithographic and electron-beam lithographic techniques [13]. Electrodes consisting of layers of $10 \mathrm{~nm}$ titanium, $10 \mathrm{~nm}$ palladium and $100 \mathrm{~nm}$ gold, with minimum feature sizes in the range $0.5 \mu \mathrm{m}$ to $5 \mu \mathrm{m}$ were fabricated. A scanning electron micrograph of a sawtooth array used for trapping of TMV is shown in Fig. 1. This particular electrode was manufactured with a $3 \mu \mathrm{m}$ gap using electron beam lithography, and it is clear from the image that the features are well defined, giving a precise field geometry. Fig. 2 shows a photograph of an electrode array, usually referred to as a polynomial electrode array, used for characterising the dielectrophoretic properties of latex beads. This electrode was manufactured using photolithography, with the central opening measuring $6 \mu \mathrm{m}$ across and a $2 \mu \mathrm{m}$ gap between neighbouring electrodes.

Typical electrode arrays measure $10 \mathrm{~mm} \times 10 \mathrm{~mm}$ and were fabricated in the centre of a microscope slide. The particle suspension $(10 \mu \mathrm{L})$ was pipetted onto the array and covered with a glass cover slip. Prior to use the electrode arrays were cleaned by repetitive washes in ethanol followed by ultra pure water. Movement of particles was observed using a fluorescence microscope (Nikon Microphot). Images were recorded using a video camera and S-VHS recorder, before digitising with a PC. Medium conductivities were measured using a Hewlett Packard 4192A Impedance Analyser in the frequency range $1 \mathrm{kHz}$ to $1 \mathrm{MHz}$ with a Sentek conductivity cell at $20^{\circ} \mathrm{C}$. The electric field was generated using an in-house manufactured frequency generator operating under computer control, giving $10 \mathrm{~V}$ peak-to-peak at frequencies up to 20 MHz. Electric field plots showing the 3-dimensional variation of field strength across a typical electrode array were obtained using the commercial finite element numerical analysis programme, Maxwell (Ansoft Corp., Pittsburgh, PA, USA).

\subsection{Latex spheres}

Fluorescent latex spheres, $282 \mathrm{~nm}$ in diameter, were purchased from Molecular Probes (Oregon, USA). They were supplied as carboxylate modified, with an average surface charge of $-3.1 \mu \mathrm{C} \mathrm{cm}{ }^{-2}$, and pre-loaded with a yellow-green fluorescent dye so that single particles could be observed by fluorescence microscopy. Bead samples for experimental use were prepared by washing and centrifuging three times in $1 \mathrm{mM}$ potassium chloride. 


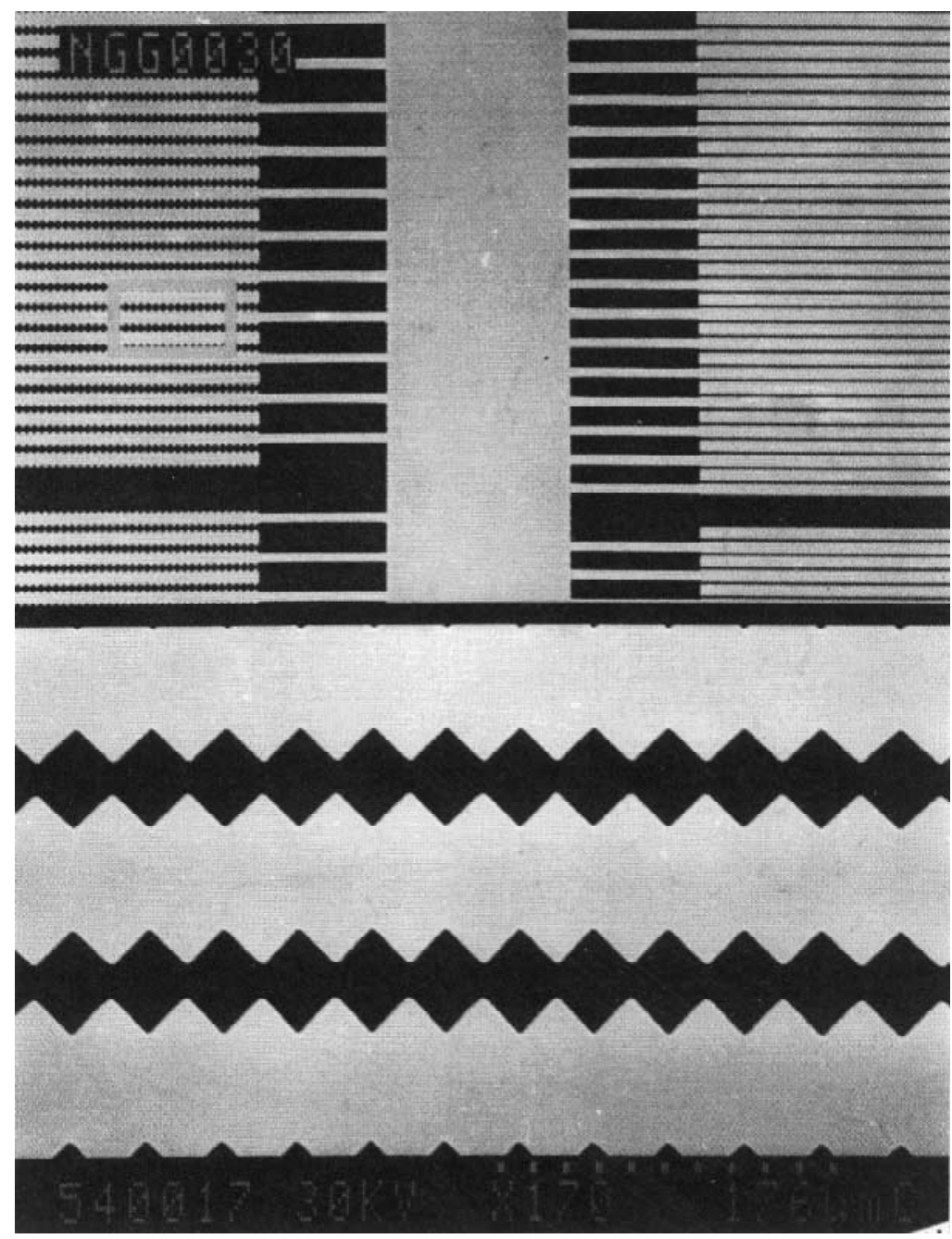

Fig. 1. Scanning electron microscope image of a sawtooth electrode array used for dielectrophoretic collection of tobacco mosaic virus. The distance between opposite electrode tips is $3 \mu \mathrm{m}$ and the total electrode area is 10 $\mathrm{mm} \times 10 \mathrm{~mm}$. The lower image is a $\times 10$ magnification of the area in the white box shown in the upper image.

\subsection{Preparation and labelling of virus}

Tobacco mosaic virus (strain U1) was raised in Nicotiana tabacum. cv. Petite Havana SR1, and purified by differential centrifugation [14]. The original inoculum was kindly donated by Prof. T.M.A. Wilson (Scottish Crop Research Institute, Invergowrie, 


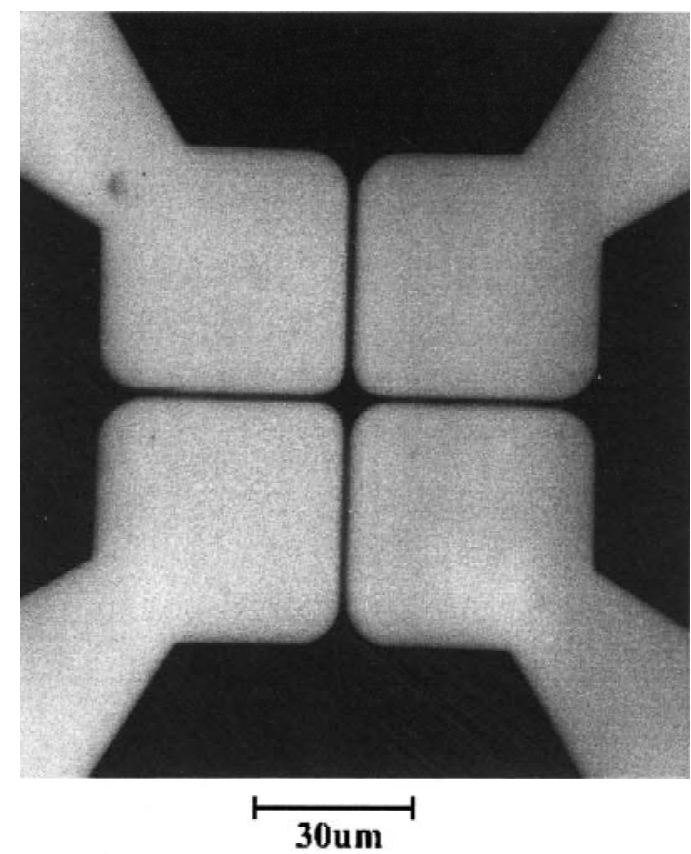

Fig. 2. A photograph of a polynomial electrode array used for trapping latex beads taken with a light microscope $(\times 60)$. The centre of the electrode is $6 \mu \mathrm{m}$ in diameter and the smallest dimension between nearest neighbour electrodes is $2 \mu \mathrm{m}$.

Scotland). Virus concentration was estimated spectrophotometrically using the published virus extinction coefficient for a $1 \mathrm{mg} \mathrm{ml}^{-1}$ solution of $E_{280}=3.0 \mathrm{mg} \mathrm{ml}^{-1}$ [15].

The virus was labelled with Rhodamine B (Sigma) by converting the fluoroprobe into an active ester, followed by direct coupling to lysine groups in the TMV coat protein [16]. The coupling procedure was carried out as follows: $48 \mathrm{mg}$ rhodamine was mixed with $11.5 \mathrm{mg} \mathrm{N}$-hydroxysuccinimide in $10 \mathrm{ml}$ water and the $\mathrm{pH}$ adjusted to $4.75 \mathrm{using}$ $0.1 \mathrm{~N} \mathrm{NaOH}$. Nine point six mg 1-ethyl-3-(3-dimethylaminopropyl)carbodiimide was added to the mixture, and the $\mathrm{pH}$ adjusted continually to 4.75 by adding $0.1 \mathrm{M} \mathrm{HCl}$. After $20 \mathrm{~min}$ the $\mathrm{pH}$ stabilised and the reaction was complete. The final mixture was then buffered with triethanolamine (TEA) to $\mathrm{pH}$ 7.2. The virus was labelled by adding excess of the rhodamine reagent $(0.5 \mathrm{ml})$ in TEA buffer, $\mathrm{pH} 7.2$ to $0.1 \mathrm{ml}$ of virus solution (at a concentration of $10 \mathrm{mg} \mathrm{ml}^{-1}$ ) and stirring overnight at room temperature. Labelled virus was separated from unreacted label by gel filtration on Sephadex G-10, and the virus collected by ultracentrifugation for $1 \mathrm{~h}$ at $200000 \times \mathrm{g}$. Labelled virus was resuspended in aliquots in potassium phosphate, $\mathrm{pH} 7.2,1 \mathrm{mM}$ EDTA. For the DEP experiments, various solution conductivities were used by varying the concentrations of potassium phosphate buffer in the range $0.1 \mathrm{mM}$ to $100 \mathrm{mM}$. 


\section{Results and discussion}

\subsection{Trapping of latex beads}

Trapping of both virus and beads was performed using appropriately sized electrode arrays. A photograph of the polynomial electrode array used for trapping the beads is shown in Fig. 2 and the electric field produced by these electrodes is shown in Fig. 3. The latter shows the field distribution in a plane through the electrode surface and demonstrates that the field is at a maximum value in the region where the electrodes are closest together and minimum in the central part of the array. In situations where the DEP force is positive, the beads should be attracted to the high field regions and held in the gaps between the electrodes. Where the DEP force is negative, the beads will be repelled from the high field regions and trapped in the centre of the electrode array. Both these effects were observed experimentally and typical results are shown in Fig. 4.

This figure shows beads undergoing positive DEP at $500 \mathrm{kHz}$ and negative DEP at 10 $\mathrm{MHz}$ at an applied voltage of $5 \mathrm{~V}$ peak to peak and in $1 \mathrm{mM}$ potassium chloride, conductivity, $14 \mathrm{mS} \mathrm{m}^{-1}$. Below $0.5 \mathrm{~V}$ no movement (other than Brownian) of the beads was observed. As the voltage was increased the beads were seen to respond to the field and move to high or low field regions depending on the applied frequency. In general the

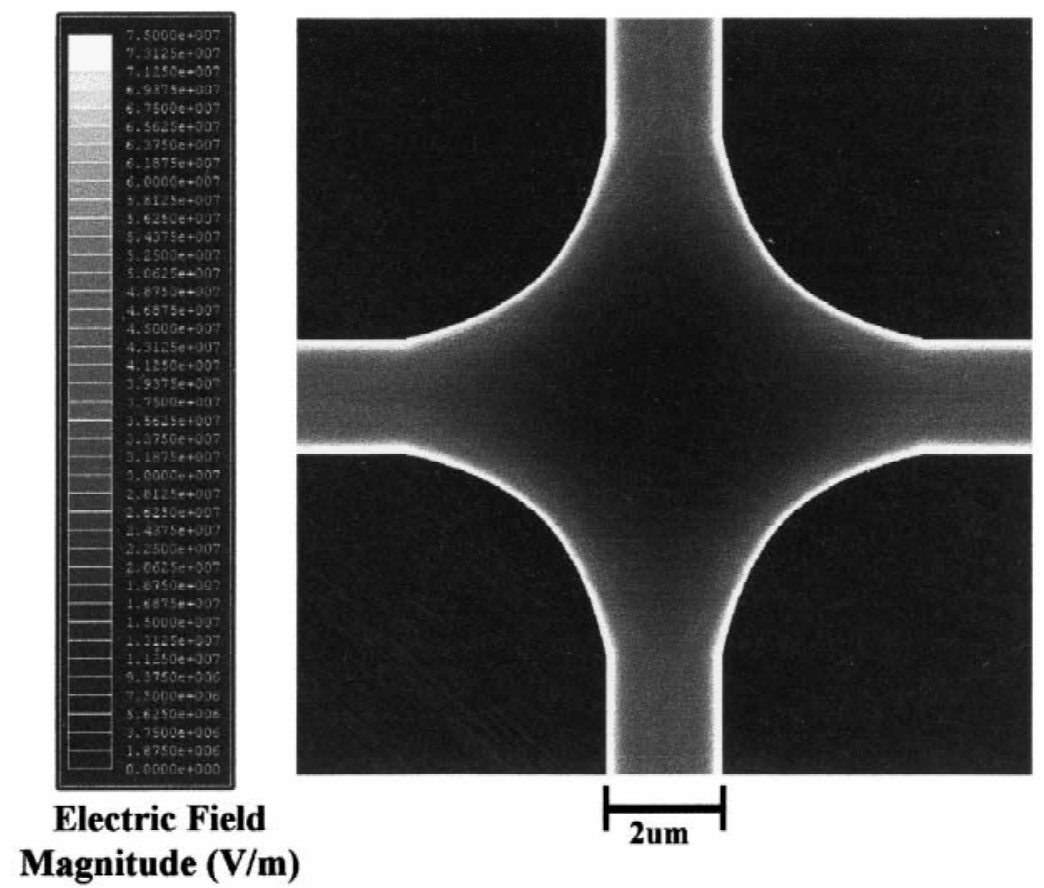

Fig. 3. The electric field produced by the electrode array shown in Fig. 2. This plot shows that the field is at a maximum in the region where the electrodes are closest together and at a minimum in the central part of the array. 


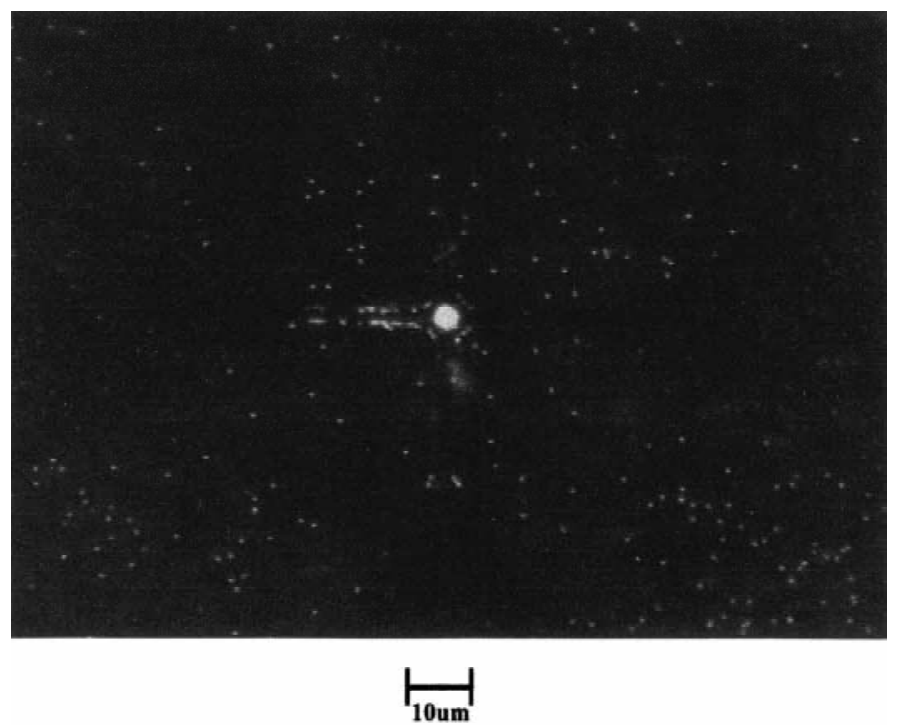

(a)

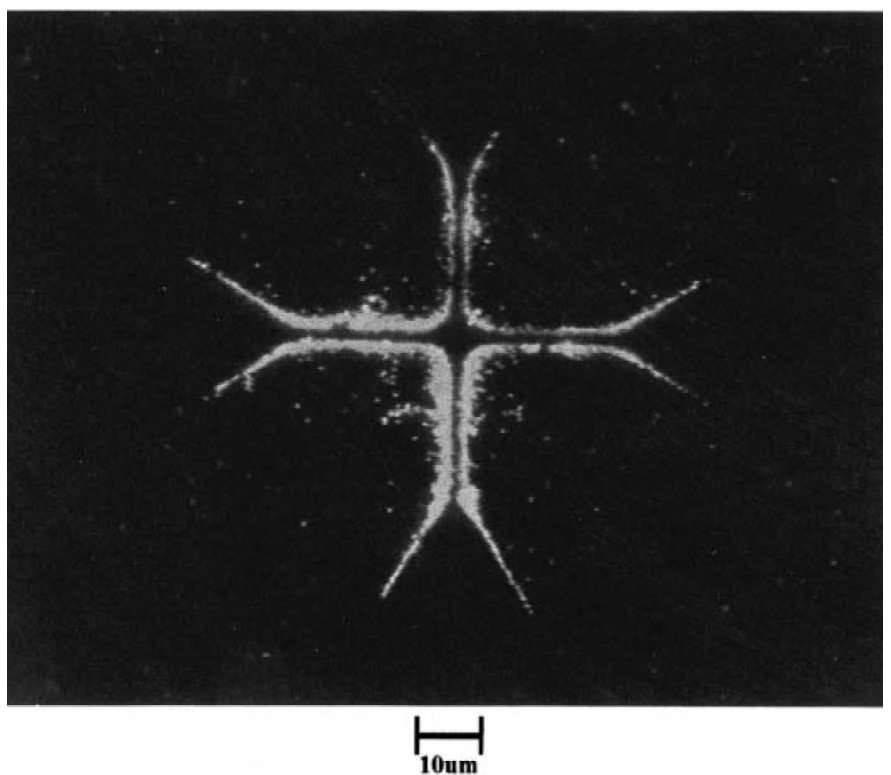

(b)

Fig. 4. Photograph showing trapping of $282 \mathrm{~nm}$ latex beads by (a) negative dielectrophoresis at a frequency of $10 \mathrm{MHz}$, and (b) positive dielectrophoresis at $500 \mathrm{kHz}$. The applied voltage was $5 \mathrm{~V}$ and the suspending medium was $1 \mathrm{mM}$ potassium chloride (corresponding to a conductivity of $14 \mathrm{mS} \mathrm{m}^{-1}$ ). 
behaviour of the beads changed from positive to negative DEP at a distinct frequency that varied depending on the conductivity (molarity) of the suspending medium. Using potassium chloride at a concentration of $1 \mathrm{mM}$ and a conductivity of $14 \mathrm{mS} \mathrm{m}{ }^{-1}$, this frequency was found to be approximately $4 \mathrm{MHz}$. The cross-over frequency was well defined and correlated with theoretical predictions concerning the known high frequency dielectric properties of latex particles ( [17], our unpublished data). Fig. 5 shows a theoretical plot of the relative force on a particle as a function of frequency and medium conductivity. The two dispersions represented were calculated using the method outlined in Zhou et al. [18] following the work of O'Brien [19]. It can be seen that as the conductivity of the medium increases, the crossover frequency decreases and the relative magnitudes of the forces change. Thus it is possible to directly control the sign and magnitude of the force on a given particle by choosing appropriate experimental conditions. The precise electrokinetic force on a particle was not calculated in this work as this depends on a number of variables such as the magnitude of the electric field gradient at the particle's precise position together with the particle volume. Other forces such as gravity, buoyancy and Brownian motion also contribute significantly to the total force on a particle.

It was possible to trap single beads for the duration of an experiment and aggregates of beads as small as $14 \mathrm{~nm}$ could be trapped by positive DEP, in agreement with the results of Muller et al. [20]. The trapping of the $282 \mathrm{~nm}$ latex beads occurred within a period of a few seconds and this period increased as the particle size decreased. Beads in the immediate vicinity of the electrodes became trapped within milliseconds and trapping of beads from outside the electrode area was a continuous process, with beads

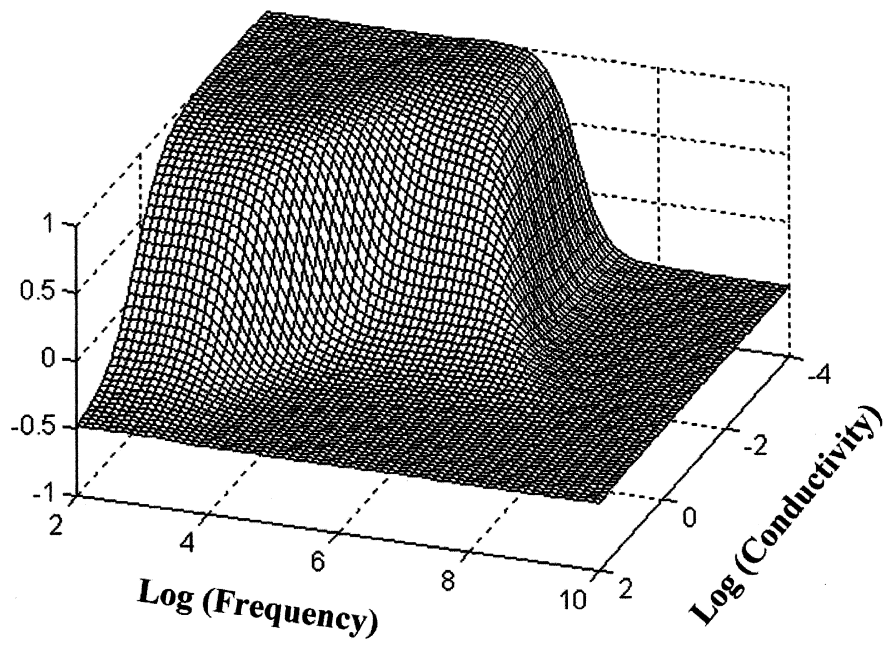

Fig. 5. Theoretical plot of the relative dielectrophoretic force on a $282 \mathrm{~nm}$ latex particle as a function of the applied frequency and medium conductivity. The plot indicates the degree and direction of electrokinetic force experienced by a bead as a function of applied field, showing quite clearly the change in sign of the force at a well defined frequency. The absolute force would also depend on parameters such as the particle size and magnitude of the local electric field gradient. 
being drawn in from the surrounding solution by hydrodynamic forces as long as the field was present. Although convection was observed at high fields and high medium conductivities this did not generally disrupt the DEP movement. The high electric fields used in these experiments would be expected to cause localised heating of the sample [20]. This in turn causes liquid streaming which carries particles into the trap, the direction and magnitude of the streaming being dependent on frequency. When the field was switched off, the beads dispersed into the surrounding medium as single entities within seconds.

The trapping of latex spheres under positive DEP at low frequencies and negative DEP at high frequencies, shows that it is possible to selectively manipulate sub-micron particles using non-uniform electric fields. The movement of particles in the electrode array is influenced by both thermal and DEP forces. The dominant electrical property of latex particles is the surface charge density and this factor controls their frequencydependent dielectrophoretic behaviour [17,21]. Any variation in the surface charge can lead to a variation in the dielectrophoretic force (both magnitude and sign). The degree of trapping depends on many parameters and consequently even beads with slightly different dielectric properties can be separated [21]. Coating beads with antibodies will directly change the surface charge properties [22] and consequently a major application of DEP could be the development of new affinity fractionation techniques.

\subsection{Trapping of tobacco mosaic virus particles}

Trapping of TMV was achieved using the sawtooth electrode array shown in Fig. 1 or also square-wave shaped electrodes (not shown). These arrays generate extremely high electric fields at the electrode tips, as shown in the electric field plot of Fig. 6. This shows the electric field magnitude for a sawtooth electrode array similar to that shown in Fig. 1, but with an inter-electrode gap of $5 \mu \mathrm{m}$. The high electric field strengths calculated for this electrode design were found to be necessary to stably trap TMV. Fig. 7 shows the trapping of TMV particles using a sawtooth electrode array at a frequency of $500 \mathrm{kHz}$, with an inter-electrode gap of $4 \mu \mathrm{m}$ and a voltage of $4 \mathrm{~V}$ (corresponding to an average field strength of $1 \times 10^{6} \mathrm{~V} \mathrm{~m}^{-1}$ ). The virus collected along the electrode edges under positive DEP, and can be seen as a white cloud between adjacent electrode tips in the figure. The fluorescence intensity was seen to continually increase with time as more virions became trapped. This occurred over the frequency range $1 \mathrm{kHz}$ to $1.5 \mathrm{MHz}$ for medium conductivities in the range $170 \mu \mathrm{S} \mathrm{cm}^{-1}$ to $14 \mathrm{mS} \mathrm{cm}^{-1}$. Above $1.5 \mathrm{MHz}$ and in this range of conductivities there was no observable trapping of the virus at the electrode edges. Occasionally large bright spots were seen and these were probably due to aggregates of virus collecting. It was not possible to resolve single TMV particles with our optical system.

The electric field induced orientation of TMV in low frequency AC fields was investigated as long ago as 1939 [23,24]. However, these studies investigated the electrophoretic effects, which depend on the fixed charge on the virus rather than the interaction of the local charges on the virus surface. In this study it has been shown that TMV can undergo positive DEP and collect at the high field regions of microelectrode structures. TMV virions comprise rod-shaped particles $300 \mathrm{~nm}$ long by $18 \mathrm{~nm}$ in 

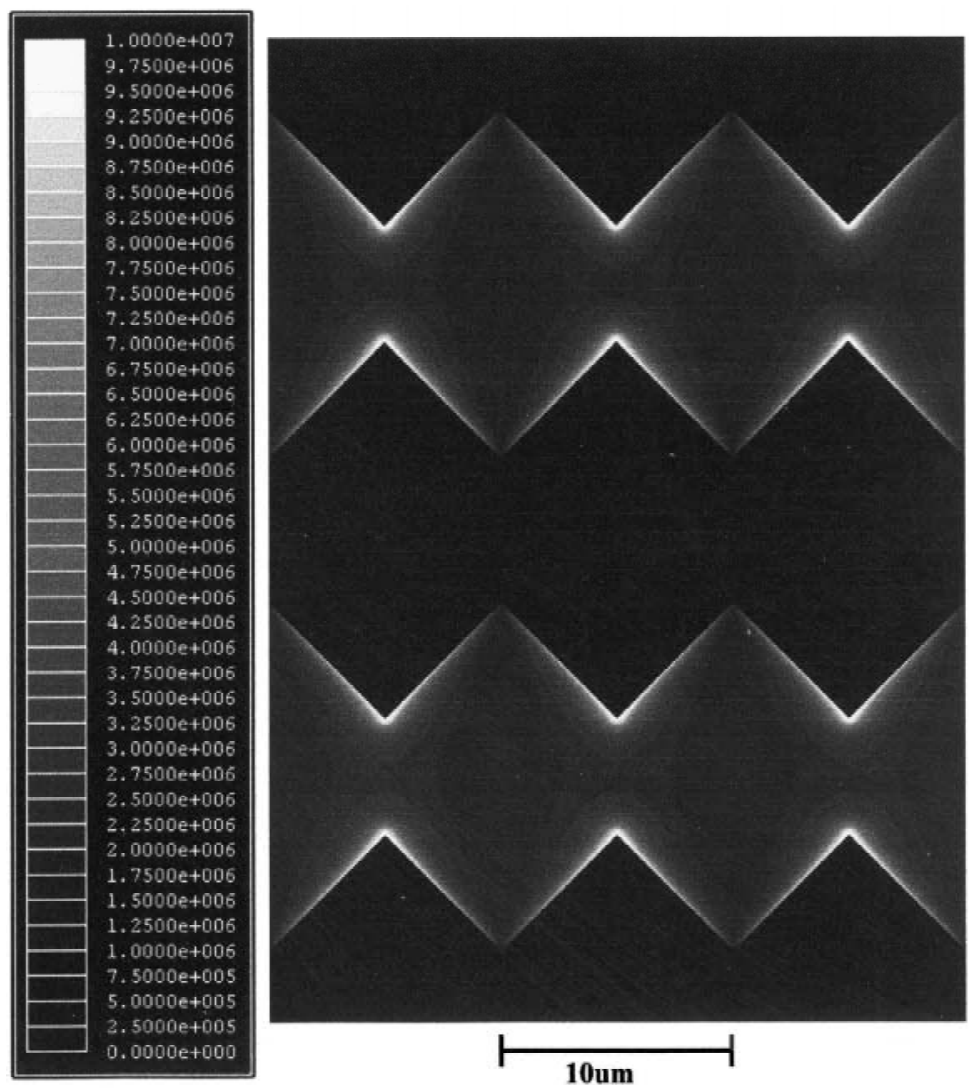

\section{Electric field magnitude $(\mathrm{V} / \mathrm{m})$}

Fig. 6. Electric field plot for the sawtooth electrode array shown in Fig. 1. The maximum field is generated at the tips and approaches $10^{8} \mathrm{~V} \mathrm{~m}^{-1}$ and corresponds to the whited regions in this plot. The minimum field strength is between the triangular electrodes, corresponding to the dark regions in the plot.

diameter [15] and this work demonstrates that dielectrophoretic trapping of such small particles can be easily achieved under the appropriate conditions. These results demonstrate that trapping of TMV is possible and are similar to the recently reported observation of the trapping of large aggregates of Sendai and Influenza virus, except that in this case viruses were trapped in so called field funnels by negative DEP [25].

The dielectrophoretic properties of a particle are governed by its dielectric properties, which in turn reflect the physiochemical makeup of the particle. For virions that have insulating lipid membranes, the dielectric properties would be expected to mirror those of cells and micro-organisms, which typically undergo negative DEP at low frequencies when the polarisability of the particle is less than the medium, and positive DEP at higher frequencies [3]. Preliminary experiments with an enveloped virus, Sonchus yellow net rhabdovirus (SYNV) [26], have demonstrated that such enveloped virions do 

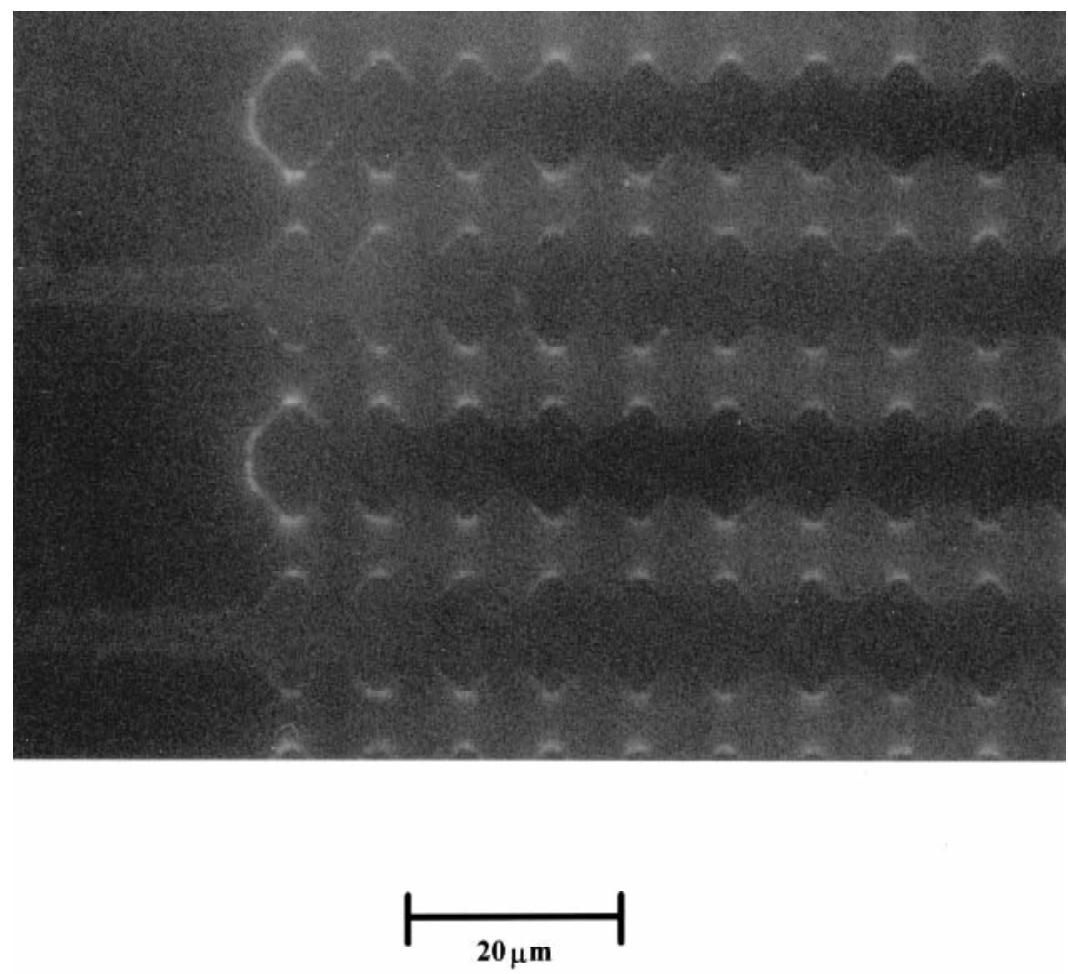

Fig. 7. Photograph showing the trapping of TMV on a sawtooth electrode array. This image shows the virus collecting in aggregates at the electrode tips as predicted by the electric field plot shown in Fig. 6. Removal of the field caused the virus to immediately disperse into the surrounding medium. Single virus particles could not be resolved and no permanent aggregation of the virus after collection was observed.

indeed qualitatively follow this DEP trend as predicted ([27], unpublished observations). In contrast, TMV does not possess an insulating membrane and consequently its polarisability will be high over a very wide frequency range and conductivity range. This is evident in the experimental results which show that for frequencies up to $1 \mathrm{MHz}$, TMV experiences positive DEP (polarisability greater than the medium). For higher frequencies, the DEP force diminishes as the polarisability of the virion reduces in magnitude, but does not differ significantly enough from the medium to give rise to a observable negative DEP force. In contrast, using an electrode array similar to that used for latex beads (Fig. 1), the enveloped virions of SYNV could be trapped by negative DEP [27], but TMV could not.

Since the DEP force depends on the polarisability of the whole TMV virion, it is to be expected that separation of similar but not identical viral particles should be achievable. For example, the replication products of several plant viruses, such as turnip yellow mosaic virus, include both RNA-containing and empty capsids [28]. In the case of other viruses like TMV, the coat protein can be re-assembled in vitro to form virus-like rod-shaped particles resembling mature infectious virions but lacking RNA [29]. As a 
consequence of the large amount of charge carried by the RNA in relation to the total charge on the virion, it might even be possible to separate RNA-containing from empty capsids.

Our experiments demonstrate that it is possible to manipulate both viruses and small latex beads by means of dielectrophoretic forces. Particles can be attracted to, or repelled from regions of different field gradient and the behaviour of the particles is consistent with simple models describing their physical makeup. The ability to manipulate viruses and sub-micron latex particles has a number of potentially rewarding biotechnological applications.

\section{Simplified description of the method and its (future) application}

The net force induced by dielectrophoresis on a collection of bioparticles depends on their dielectric properties and volume. Depending on the frequency this force can be positive (directed towards high field regions), negative (directed away from high field regions) or zero. Many biotechnological applications are now beginning to emerge from the early pioneering work using cells and micro-organisms. Scaling the technology down in size for the selective manipulation of sub-micron particles requires that the force must be increased; this can only be achieved by increasing the electric field. However, with comparatively large electrode structures, this inevitably leads to the generation of large amounts of heat within the device. The temperature rise in any given electrode geometry can be minimised by making the active area of the electrodes as small as possible, therefore by using micro-electrode arrays it is possible to generate the required electric field gradients without a concomitantly large increase in power dissipation. Furthermore, by maintaining a high surface to volume ratio there is a more rapid dissipation of any heat generated in the array. The fabrication of microelectrode structures using semiconductor technology, has meant that such structures can now be reliably made with $\mu \mathrm{m}$ scale dimensions. As a consequence, large-scale power dissipation is greatly reduced. Using these micro-electrodes it is possible to manipulate aggregates and even single biological particles in the sub-micrometre range. A potentially more sensitive and rapid separation technology is emerging with applications in a wide range of technologies from diagnostics to new affinity fractionation systems.

\section{Acknowledgements}

We thank Prof. J.R. Coggins and Ms Sharon Hardy for assistance with labelling the TMV, and the EPSRC for a studentship for N.G. Green. We also acknowledge the Biotechnology and Biological Sciences Research Council (Grant No: 17/T05315) for funding part of this work. We thank Dr J.P.H. Burt, University of Wales, Bangor, for assistance with electric field plotting. 


\section{References}

[1] Pohl HA. The motion and precipitation of suspensoids in divergent electric fields. J Appl Phys 1951;22:869-71.

[2] Pohl HA. Dielectrophoresis, Cambridge University Press, Cambridge U.K., 1978.

[3] Pethig R. Dielectrophoresis: Using inhomogeneous A.C. electric fields to separate and manipulate cells. Crit Rev Biotechnol 1996;16:331-48.

[4] Markx GH, Huang Y, Zhou XF, Pethig R. Dielectrophoretic Characterisation and Separation of Micro-organisms. Microbiology-UK 1994;140:585-91.

[5] Markx GH, Talary MS, Pethig R. Separation of viable and non-viable yeast using dielectrophoresis. J Biotechnology 1994;32:29-37.

[6] Burt JPH, Pethig R, Gascoyne PRC, Becker FF. Dielectrophoretic characterisation of Friend murine erythroleukaemic cells as a measure of induced-differentiation. Biochim Biophys Acta 1990;1034:93101.

[7] Gascoyne PRC, Noshari J, Becker FF, Pethig R. Use of dielectrophoretic collection spectra for characterising differences between normal and cancerous cells. IEEE Trans Ind Appl 1994;30:829-34.

[8] Becker FF, Wang X-B, Huang Y, Pethig R, Vykoukal J, Gascoyne PRC. Separation of human breast cancer cells from blood by differential dielectric affinity. Proc Natl Acad Sci USA 1995;92:860-4.

[9] Talary MS, Mills KI, Hoy T, Burnett AK, Pethig R. Dielectrophoretic separation and enrichment of CD34 + cell subpopulation from bone marrow and peripheral-blood stem-cells. Med Bio Eng Comp 1995;33:235-7.

[10] Mills KI, Stephens M, Talary M, Pethig R, Burnett AK. Dielectrophoretic separation of CD34-positive cells from peripheral stem-cells harvests. Blood 1995;86:462.

[11] Stephens M, Talary MS, Pethig R, Burnett AK, Mills KI. The dielectrophoresis enrichment of CD34( + ) cells from peripheral blood stem-cells harvests. Bone Marrow Transplant 1996;18:777-82.

[12] Wang X-B, Huang Y, Burt JPH, Markx GH, Pethig R. Selective dielectrophoretic confinement of bioparticles in potential-energy wells. J Phys D: Appl Phys 1993;26:1278-85.

[13] Morgan H, Green NG, Hughes MP, Monaghan W, Tan TC. Large-area travelling wave dielectrophoresis particle separator. J Micromech Microeng 1997;7:65-70.

[14] Boedker H, Simmons NS. Preparation and characterisation of essentially uniform tobacco mosaic virus particles. J Am Chem Soc 1958;80:2550-6.

[15] Zaitlin M, Israel HC. CMI/AAB Descriptions of Plant Viruses, Number 151, 1975.

[16] Mathews REF. Plant Virology Academic Press Incorporated, London Ltd, 1981.

[17] Arnold WM, Schwan HP, Zimmerman U. Surface conductance and other properties of latex particles measured by electrorotation. J Phys Chem 1987;91:5093-8.

[18] Zhou X-F, Markx GH, Pethig R, Eastwood IM. Differentiation of viable and non-viable bacterial biofilms using electrorotation. Biochim Biophys Acta 1995;1245:85-93.

[19] O-Brien RW. The high-frequency dielectric dispersion of a colloid. J Colloid Int Sci 1986;113:81-93.

[20] Muller T, Gerardino A, Schnelle Th, Shirley SG, Bordoni F, De Gasperis G, Leoni R, Fuhr G. Trapping of micrometre and sub-micrometre particles by high-frequency electric fields and hydrodynamic forces. J Phys D: Appl Phys 1996;29:340-9.

[21] Green NG, Morgan H. Dielectrophoretic Separation of Nano-particles. J Phys D: Appl Phys 1997;30:L41-44.

[22] Burt JPH, Chan KL, Dawson D, Parton A, Pethig R. Assays for Microbial Contamination and DNA Analysis based on Electrorotation. Ann Bio Clin 1996;54:253-7.

[23] O'Konski CT, Zimm BH. New Methods for studying electrical orientation and relaxation effects in aqueous colloids: Preliminary results with Tobacco Mosaic Virus. Science 1950;111:113-6.

[24] Lauffer MA. Electro optical effects in certain viruses. J Am Chem Soc 1939;61:2412-6.

[25] Muller T, Fiedler S, Schnelle T, Ludwig K, Jung H, Fuhr G. High Frequency Fields for Trapping of Viruses. Biotechnol Tech 1996;10:221-6.

[26] Jackson AO, Christie SR. Purification and some physicochemical properties of Sonchus yellow net virus. Virology 1977;77:344-55. 
[27] Green NG, Morgan H, Wilkinson CDW, Milner JJ. Dielectrophoresis of virus particles, Proceedings of the St Andrews Meeting of the SEB UK, C220, 1994, p 77.

[28] Huxley HE, Zubay G. Structure of the protein shell of turnip yellow mosaic virus. J Mol Biol 1960;2:189-96.

[29] Richards KE, Williams RC. Assembly of tobacco mosaic virus in vitro. Comp Virology 1976;6:1-37. 